EDUCACIÓN

Julio - Diciembre. 2019, Vol. 25 N²: pp. 175-192

DOI: 10.33539/educación.2019.v25n2.2046

\title{
AUTOLESIÓN: CICATRIZANDO ALMAS
}

\section{INTERVENTION PROGRAM THE CUTTING: HEALING SOULS}

\author{
Janis Belletich Sandoval \\ janisbelletich@gmail.com \\ Débora Céspedes Castañeda \\ debycescas123@gmail.com \\ Nélida Díaz Mego \\ diazmegonelida@gmail.com \\ Milagros Pumapillo Rosas \\ Isabelrosas1002@gmail.com
}

\begin{abstract}
RESUMEN
Este artículo plantea un programa de intervención y detalla el abordaje de los estudiantes que practican "cutting", cuál es el rol y participación de los padres de familia y cómo influye este accionar en su prevención. Incluyen actividades que se pretenden ejecutar para fortalecer las habilidades, cualidades y destrezas de los estudiantes, afectados y no afectados en las diferentes áreas.
\end{abstract}

\section{PALABRAS CLAVE}

Cutting, prevención, abordaje, padres de familia y estudiantes.

\section{ABSTRACT}

This article proposes an intervention program and describes the addressing of the students who practice "Cutting", what the parents' roles and participation are and how this influences in its prevention. Furthermore, it includes activities that strengthen the skills, qualities and abilities in affected and non-affected students that involve different subjects at school.

\section{KEYWORDS}

Cutting, prevention, addressing, parents and students.
$\mathrm{E}$ presente artículo tiene como finalidad dar a conocer "El cutting", que se manifiesta como una práctica recurrente en los adolescentes en la actualidad, y la que se viene convirtiendo en una problemática social en las escuelas de nuestro país.

Todos conocemos que los adolescentes se encuentran en una etapa compleja de su desarrollo, sobre todo en el plano emocional, pues, se hallan definiendo su personalidad y donde consolidan aprendizajes que los guiarán en su vida adulta. Es en esta etapa que se presentan alteraciones conductuales que llegan a interferir en su desempeño y desenvolvimiento social, que deben tratarse oportunamente evitando, de esta manera, que luego se conviertan en problemas y si no hay un abordaje pasen a ser patologías.

Este artículo plantea un programa de intervención y detalla cómo debe ser el abordaje de los estudiantes que practican "cutting", cuál es el rol 
y participación de los padres de familia y cómo influye este accionar en su prevención.

Además, se detallan las actividades que se pretende ejecutar para fortalecer las habilidades, cualidades y destrezas de los estudiantes, afectados y no afectados, de manera conjunta con las áreas de: Tutoría, Educación Cívica, Religión (Pastoral) y Personal Social.

Nuestra población de estudio fueron todos los estudiantes de primero a quinto de secundaria de una entidad parroquial de Lima. El fin de esta investigación es que se conozca y se plantee un modelo de abordaje como prevención para los estudiantes de otras instituciones.

\section{PROGRAMA DE INTERVENCIÓN}

\section{DEFINICIÓN DEL TEMA}

En las últimas décadas se ha puesto en boga una práctica común entre los adolescentes, que consiste en emplear objetos cortantes o punzantes con la finalidad de infringirse dolor y/o autolesión (autoagredirse). La consulta es ¿Por qué toman esa determinación? o ¿Cuáles son las razones para autolesionarse? ¿Por qué surge de repente este fenómeno?

La autolesión se asocia generalmente con personas de personalidad retraída, tímidas, sensibles y en su mayoría, depresivas. Al no poder expresar sus emociones no encuentran otra forma de desahogar su frustración mediante una práctica silenciosa como es el "cutting", estas autolesiones suelen realizarse en zonas poco visibles como muñecas, brazos y piernas, pues, logran cubrir las lesiones con prendas de vestir holgadas, vendaje o maquillaje.

Para Cruz (2016) el "cutting" lo define como: "la acción lesiva intencional que una persona lleva a cabo en sí misma, produciéndose con ello un daño corporal de baja letalidad, de una naturaleza socialmente inaceptable; se trata de una acción deliberada y comúnmente repetitiva", la cual puede implementarse de forma crónica como una forma de reducir el estrés o malestar emocional; es decir, un mecanismo de enfrentamiento autodestructivo.

Según los datos emitidos a raíz del informe ejecutado por la Fundación Camelot y Fundación de la Salud Mental, se evidencia autolesiones en un porcentaje de niños, algunos adultos, pero, sobre todo, entre la población adolescente y juvenil con edades comprendidas entre los $15 \mathrm{y}$ 25 años, además uno de cada 5 jóvenes ingresa en las tasas de autolesión en Reino Unido, lugar con mayor índice en Europa (Brophy \& Holmstrom, 2006).

Se establece como causa principal del "Cutting" a la violencia intrafamiliar, siendo el seno familiar el encargado de la contención emocional y al no tener este soporte todo el sistema familiar y personal se resquebraja, la convivencia en armonía se pierde dando partida a una convivencia desagradable llena de gritos, ofensas, peleas, desgastando así los valores tan importantes en este contexto como: el respeto, confianza, honestidad, amor, pertenencia, autoestima, compromiso; sumado a ello la falta de comunicación ocasiona situaciones violentas que acarrean la ruptura de relaciones interpersonales $\mathrm{y}$, con ello, una inadecuada formación y desarrollo en los hijos, pues, al estar inmersos dentro de constantes maltratos existe la posibilidad que en un futuro se dé paso a actitudes transgeneracionales; es decir, la repetición de patrones, lo que lleva a una disfunción familiar en la que sus roles se vean alterados.

\section{PLANTEAMIENTO DEL PROBLEMA}

Las instituciones educativas al inicio de la modernidad y con los cambios de la globalización vienen presentando problemas de orden emocional y psicológico por parte de sus estudiantes.

Una de las principales dificultades es el manejo de las emociones, lo que desencadena a una serie de patologías como son: los desórdenes alimenticios (anorexia y bulimia), depresión y cutting. 
Las conductas autolesivas (cutting) consisten en realizarse daño físico, sin intención suicida. Hay una amplia diversidad clínica de este grupo de comportamientos y a nivel psíquico se considera que estas conductas de daño son un método inconsciente de expresión y alivio al sufrimiento psicológico; por lo tanto, pueden en un momento considerarse como un síntoma predictor de un acto suicida.

Estas conductas se presentaron en un inicio en países europeos; sin embargo, se vienen evidenciando también en Estados Unidos y Sudamérica, por ello hay que tener cuidado con el uso de la tecnología ya que este es el medio por el cual, con ejemplos, ya sea de blogueros, youtubers, canales de instragram y Facebook (tendencias sociales), se comparte la información.

Durante los últimos años las incidencias de cutting han ido en aumento en los alumnos de 3ro. a 5to. año de secundaria, por lo que nos vemos en la necesidad de hacer algo para remediar tal dificultad.

Al preguntarles a nuestros alumnos por qué realizan dichas acciones, todos ellos manifiestan que es un comportamiento intencional el herirse a sí mismo, haciéndose daño físico en su propio cuerpo, con lo que se intenta cambiar el estado de ánimo, en donde se da la sensación de irrealidad y de vacío, al producirse un adormecimiento de sus emociones. Estas conductas les sirven a las personas como mecanismo de autorregulación para una mejor adaptación dentro del entorno.

La mayoría de los casos se presenta en adolescentes de entre 10 y 16 años, de acuerdo con lo mencionado por Emilia Gómez, experta universitaria y lo que organizaciones internacionales indican que la edad promedio en la que comienza a registrarse esa conducta es a los 12 años.

La mayoría de nuestros alumnos esconden problemas mucho más graves detrás de las autolesiones y es que se sabe que el cutting es un desfogue para los estudiantes, sin saber los peligros que experimentan.

A pesar de contar con un equipo de apoyo y orientación psicológica, consideramos que aún no hemos podido solucionar y revertir el problema, es por ello que, con la elaboración de nuestro programa de intervención y la participación del docente para detectar los casos en nuestros alumnos, se realizará el seguimiento pertinente.

\section{POBLACIÓN}

La población de estudio estuvo constituida por estudiantes de 1ro. a 5to. año del nivel secundario de la Institución Educativa Pública de Lima, durante el periodo de abril, mayo y junio. Como se observa en el cuadro: 1ro. A de secundaria está integrada por 14 mujeres y 19 varones; 1ro. B, 12 mujeres y 20 varones; 2 do. A, 12 mujeres y 18 varones; 2do. B, 17 mujeres y 13 varones; 3ro. A, 15 mujeres y 16 varones; 3ro. B, 19 mujeres y 11 varones; 4to. A, 17 mujeres y 15 varones; 4to. B, 18 mujeres y 14 varones; 5to. A, 18 varones y 17 mujeres; 5to. B, 21 mujeres y 14 varones, formando un total de 323 estudiantes. De la misma manera, de acuerdo a lo que se ha observado, hay en total 12 estudiantes que presentan conductas autolesivas, y todas son alumnas de tercero a quinto de secundaria. 


\begin{tabular}{|c|c|c|c|c|}
\hline & Grado & Mujeres & Varones & Total \\
\hline 1 & Primero A & 14 & 19 & 35 \\
\hline 2 & Primero B & 12 & 20 & 32 \\
\hline 3 & Segundo A & 12 & 18 & 30 \\
\hline 4 & Segundo B & 17 & 13 & 30 \\
\hline 5 & Tercero A & 15 & 16 & 31 \\
\hline 6 & Tercero B & 19 & 11 & 31 \\
\hline 7 & Cuarto A & 17 & 15 & 32 \\
\hline 8 & Cuarto B & 18 & 14 & 32 \\
\hline 9 & Quinto A & 18 & 17 & 35 \\
\hline 10 & Quinto B & 21 & 14 & 35 \\
\hline & \multicolumn{3}{|c|}{ Total de estudiantes } \\
\hline
\end{tabular}

\section{ANÁLISIS DE LA SITUACIÓN}

En una Institución Educativa del distrito de Ate, Urbanización Salamanca, se evidencia una problemática escolar en adolescentes del nivel secundaria de 3ro. a 5to. año, de las cuales hemos identificado en total 12 estudiantes que presentan conductas autolesivas. Los docentes manifestaron que varias de ellas provienen de familias disfuncionales, madres solteras y jóvenes que aún no han dejado de ser hijas, padres permisivos, lo cual las expone a peligros sociales como consumo de alcohol y sustancias ilegales como las drogas, además, algunos problemas de conducta, entre otros.

La situación escolar de dicha institución educativa, al parecer es mejor que otras instituciones de los alrededores, por lo cual la mayoría de estudiantes reciben atención psicológica para ayudarlos a resolver sus problemas en el aspecto físico, mental $\mathrm{y}$ emocional; también cuentan con el apoyo de una licenciada para la atención de primeros auxilios en casos de emergencia.

De la misma manera, según los informes que hemos podido observar tanto de psicología, tutoría y departamento de pastoral, las complicaciones de algunos problemas se deben a la falta de apoyo y atención debida de parte de los padres de familia que no siguen las indicaciones y sugerencias que les brinda cada área y, en general, la institución. Las actitudes de los padres de las estudiantes con conductas autolesivas, la mayoría es por la falta de comunicación con sus menores hijos o simplemente no asisten cuando se les cita en los días indicados por cada área.

Asimismo, se ha podido descartar algunos problemas que comprometen a los padres, como: la falta de tiempo de calidad, comunicación, violencia a través de maltrato físico y psicológico. Todos estos aspectos mencionados, probablemente se encuentren asociados a una conducta inesperada que es la autolesión como un mecanismo compensador.

Por ello, el presente programa de intervención contribuirá, para previsión e implementación de contenidos educativos sobre conductas autolesivas en adolescentes, con temas dirigidos a los profesores, padres de familia de la institución, de tal manera que se tenga una conducta vigilante y se pueda ayudar anticipadamente. Asimismo, consideramos que este programa de intervención favorecerá al personal de enfermería, enfatizando su rol en promoción de salud escolar y comunitaria en el adolescente, que permita alcanzar un estilo de vida saludable. 


\begin{tabular}{|l|c|c|c|}
\hline & & \multicolumn{3}{|c|}{ Número de adolescentes afectadas (os) } \\
\hline & Mujeres & Varones & Grados \\
\hline 1 & 0 & 0 & Primero A \\
\hline 2 & 0 & 0 & Primero B \\
\hline 3 & 0 & 0 & Segundo A \\
\hline 4 & 0 & 0 & Segundo B \\
\hline 5 & 2 & 0 & Tercero A \\
\hline 6 & 1 & 0 & Tercero B \\
\hline 7 & 1 & 0 & Cuarto A \\
\hline 8 & 3 & 0 & Cuarto B \\
\hline 9 & 3 & 0 & Quinto A \\
\hline 10 & 2 & 0 & Quinto B \\
\hline & 12 & 0 & 10 secciones participantes \\
\hline
\end{tabular}

\section{Árbol de problemas}

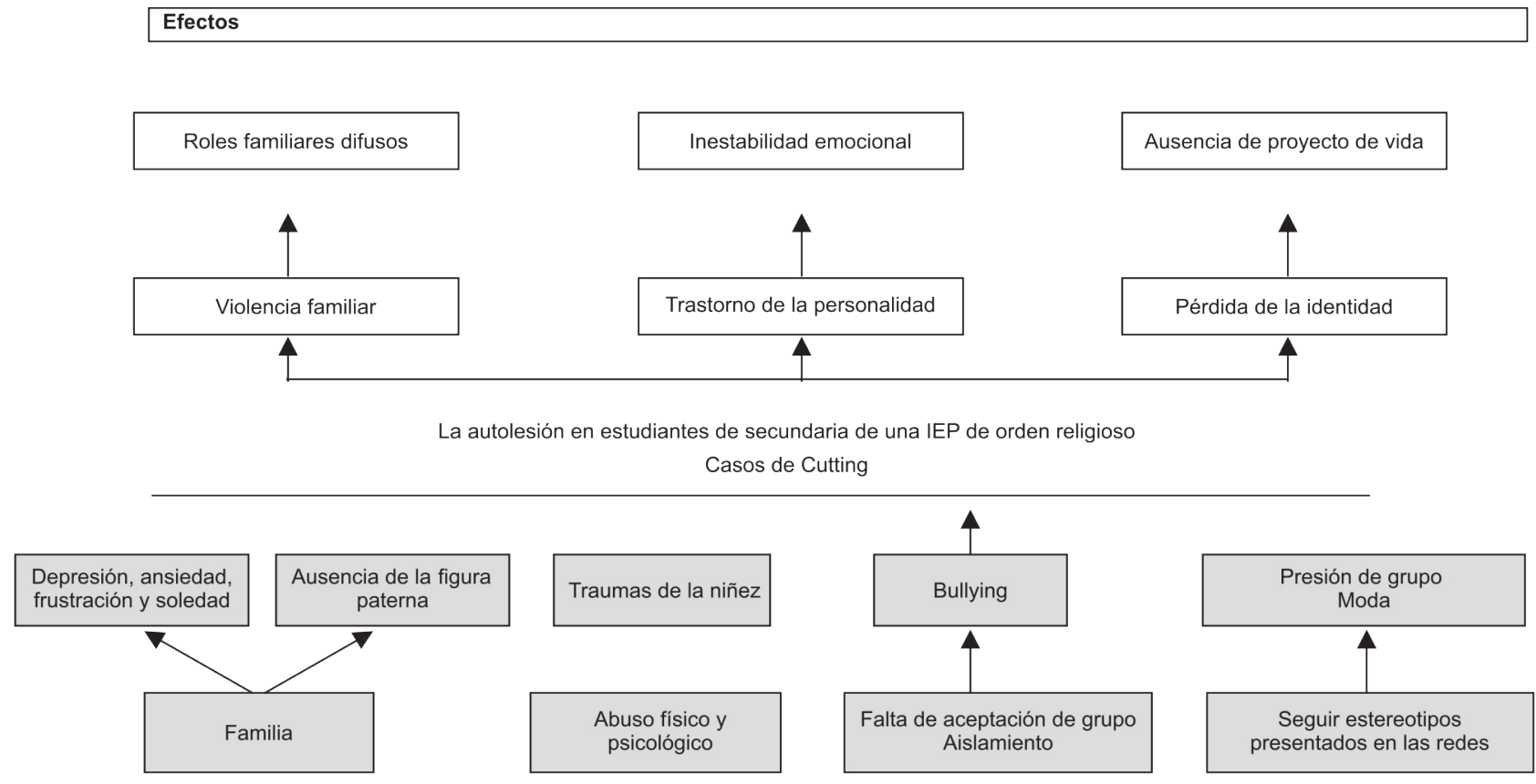

\section{OBJETIVO}

\subsection{Objetivo General}

Ejecutar el programa de intervención "Cicatrizando almas" para ayudar a las estudiantes con problemas de autolesión.

\subsection{Objetivos específicos}

a. Identificar las causas de las autolesiones de las estudiantes a través del programa de intervención.

b. Informar y orientar a los padres de familia sobre los hechos ocurridos con sus menores hijas que se han autolesionado.

c. Realizar sesiones de sensibilización e información sobre las causas, consecuencias y prevención de las autolesiones. 
d. Prevenir a la comunidad educativa sobre los problemas que pueden emerger durante la adolescencia.

\section{ACTORES}

El programa se ejecutará con la participación de los siguientes actores:

\begin{tabular}{|l|l|}
\hline \multicolumn{1}{|c|}{ Actores } & \multicolumn{1}{c|}{ Participación } \\
\hline Alumnas afectadas & $\begin{array}{l}\text { Estudiantes de 1ro. a 5to. de secundaria entre edades de 13 a 17 años, que serán } \\
\text { beneficiarias del programa de intervención. }\end{array}$ \\
\hline Tutores & $\begin{array}{l}\text { Encargados de las sesiones de sensibilización e información del tema de } \\
\text { autolesión. }\end{array}$ \\
\hline Padres de Familia & $\begin{array}{l}\text { Recibirán charlas de información y orientación sobre el problema. Cabe } \\
\text { mencionar que va dirigido a los padres de las menores afectadas y en general. }\end{array}$ \\
\hline Psicólogo & $\begin{array}{l}\text { Sesiones con las menores afectadas y sus padres. } \\
\text { Apoyo en las sesiones realizadas por el tutor. } \\
\text { Charlas a los padres de familia sobre el problema. }\end{array}$ \\
\hline
\end{tabular}

\section{ESTRATEGIAS}

En la institución del estudio se ha observado que 12 alumnas se vienen ocasionando autolesiones en diferentes partes del cuerpo.

Para ello, se ha elaborado un Programa de intervención que pretende ayudar a los estudiantes $y$, a la vez, concientizar al resto de sus compañeros para que no realicen los mismos actos.

Definiremos diferentes grupos con los que se realizarán diversas actividades.

1. Alumnas afectadas, son las alumnas que realizan las autolesiones.

2. Compañeras y compañeros de las alumnas afectadas, son las personas más cercanas a imitar la conducta o ayudar a sus compañeras afectadas.

3. Padres de Familia de las alumnas afectadas.

4. Padres de Familia de 1ro. a 5to. de Secundaria.

Es importante rescatar que este programa de intervención recibirá el apoyo de toda la comunidad educativa con el fin de brindar una solución inmediata y lograr el éxito del programa.

En cuanto a las estrategias que se utilizarán son software en las diferentes actividades.
A continuación, se detalla cada actividad.

Nombre de la Propuesta: "Yo me cuido, protejo y amo".

\section{Diseño de la propuesta de intervención}

Teniendo en cuenta la problemática, se propone intervenir en cuatro áreas específicas de trabajo:

1. Personas afectadas, es decir, alumnas que se han inflingido cortes.

2. Familiares de personas afectadas.

3. Personas y alumnos no afectados, los mismos que se convierten en una población de riesgo y vulnerable según el comportamiento social en el que todo se convierte en moda y por aprobación social, cometen actos peligrosos y hasta delictivos.

4. Familiares de personas no afectadas.

Materias que se pueden articular para el tratamiento de la problemática

El contexto en el que se desarrollará la propuesta de intervención se encuentra dentro de las aulas de secundaria de un colegio privado de Lima, siendo esta una institución educativa religiosa hemos tenido 
en cuenta articular varias materias a fin de poder incidir permanentemente en el cambio.

- Tutoría.

- Educación Cívica.

- Religión (Pastoral).

- DPCC.

Asimismo, y siendo conscientes que estamos tratando un problema exclusivamente psicológico, debemos contar con un psicólogo especialista en el área, pues será él quien determine la viabilidad de cada acción o medida a ejecutar. Por ello, hemos incluido a diversos profesionales en este plan de intervención:

- Psicólogo especialista en el área.

- Psicólogo del colegio.

- Profesores y plana directiva.

- Alumnos.

- Familiares de alumnos.

- Trabajadora Social.

- Enfermera de la Institución Educativa.

Debido a esta división se proponen actividades separadas para cada grupo, en sesiones de estudiantes afectados, no afectados y padres de familia.

\section{ACTIVIDADES PARA EL TRABAJO CON ALUMNAS AFECTADAS}

\begin{tabular}{|c|c|c|}
\hline \multicolumn{3}{|c|}{ ACTIVIDADES Y ESTRATEGIAS DE PROGRAMA DE INTERVENCIÓN } \\
\hline SESIÓN 1 & LIBERA TUS EMOCIONES & EVALUACIÓN \\
\hline $\begin{array}{l}\text { DURACIÓN: } \\
2 \text { horas }\end{array}$ & $\begin{array}{l}\text { MOMENTOS: } \\
\text {-INICIO } \\
\checkmark \text { Observa y escucha el video como motivación: https://www.youtube.com/ } \\
\text { watch?v=-VFiDEgW0mM } \\
\checkmark \text { Responde las siguientes preguntas: ¿Qué es lo que sentimos al ver el video? } \\
\text { ¿Qué emociones observamos en el video? } \\
\checkmark \text { Tomando como referencia sus respuestas se vincula con el tema Control de } \\
\text { Emociones. } \\
\text {-PROCESO } \\
\checkmark \text { Dinámica: nos colocamos todos de pie y formamos un círculo con las sillas. } \\
\text { La psicóloga especialista pedirá que todas cierren los ojos y realizará } \\
\text { preguntas y las personas que pasan por la situación que está nombrando la } \\
\text { psicóloga, se quedarán de pie. } \\
\checkmark \text { Las preguntas que se realizarán son: } \\
\text { ¿Alguna vez te has sentido sola? } \\
\text { ¿Quién alguna vez perdió a un ser querido? } \\
\text { ¿Alguna vez has sentido que nadie te ama? } \\
\text { ¿A quién la dejó el enamorado? } \\
\text { ¿Sientes que no eres importante para tu familia? } \\
\text { ¿Quién ha sufrido maltrato psicológico o físico? } \\
\checkmark \text { Luego escribiremos frases de apoyo a la compañera para que se sienta } \\
\text { mejor. } \\
\checkmark \text { Se entregará un chocolate a cada participante. } \\
\checkmark \text { Se utilizará la app Termotic que, además de identificar las emociones básicas, } \\
\text { permite indicar la intensidad de lo que sienten, permitiendo la posibilidad de } \\
\text { crear un diario personal en el que registrar las propias emociones. } \\
\text { OBSERVACIONES: De acuerdo con lo que determine la psicóloga, se realizará } \\
\text { el monitoreo de las alumnas. } \\
\checkmark \text { Se realizará la retroalimentación sobre lo que se ha observado. } \\
\text { Se enseñará una técnica de relajación como cierre. } \\
\text { OLA }\end{array}$ & 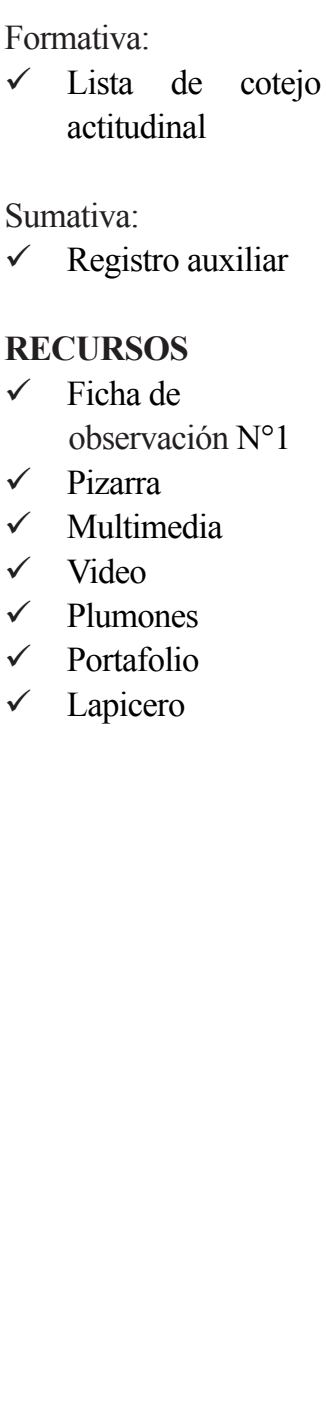 \\
\hline
\end{tabular}




\section{ACTIVIDADES Y ESTRATEGIAS DE PROGRAMA DE INTERVENCIÓN}

\begin{tabular}{|c|c|c|}
\hline $\begin{array}{l}\text { SESIÓN } 2 \\
\text { DURACIÓN: } \\
2 \text { horas }\end{array}$ & 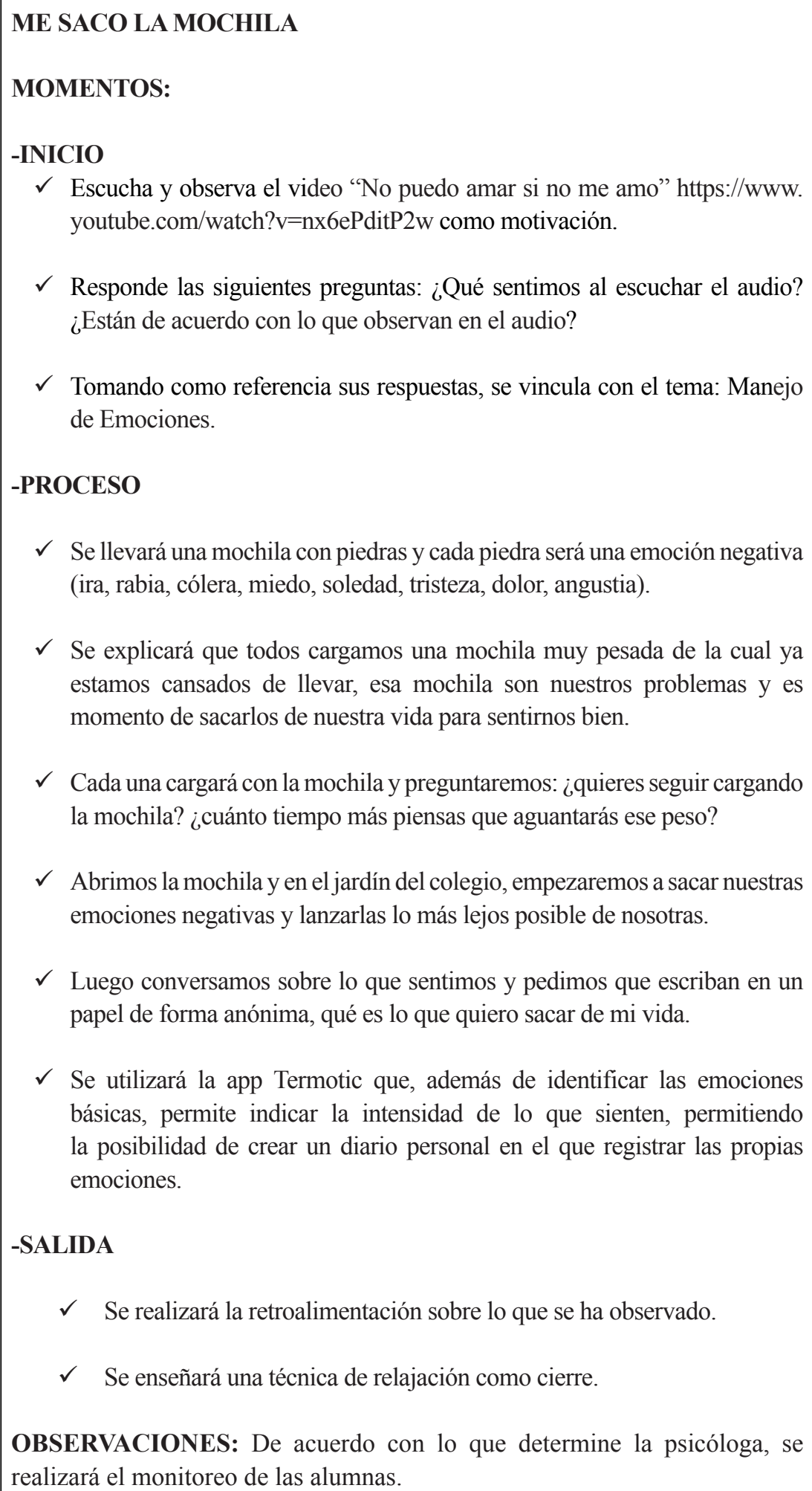 & $\begin{array}{ll}\text { EVALUACIÓN } \\
\text { Formativa: } \\
\checkmark & \text { Lista de cotejo } \\
& \text { actitudinal } \\
\text { Sumativa: } & \\
\checkmark & \text { Registro auxiliar } \\
\text { RECURSOS } \\
\checkmark & \text { Ficha de trabajo } \\
& \text { No } 1 \\
\checkmark & \text { Pizarra } \\
\checkmark & \text { Multimedia } \\
\checkmark & \text { Video } \\
\checkmark & \text { Plumones } \\
\checkmark & \text { Portafolio } \\
\checkmark & \text { Lapicero }\end{array}$ \\
\hline
\end{tabular}




\begin{tabular}{|c|c|c|}
\hline \multicolumn{3}{|c|}{ ACTIVIDADES Y ESTRATEGIAS DE PROGRAMA DE INTERVENCIÓN } \\
\hline $\begin{array}{l}\text { SESIÓN } 3 \\
\text { DURACIÓN: } \\
2 \text { horas }\end{array}$ & $\begin{array}{l}\text { ¿QUIÉN ERES EN REALIDAD? } \\
\text { MOMENTOS: } \\
\text {-INICIO: } \\
\checkmark \quad \text { Escucha y observa el video https://www.youtube.com/ } \\
\text { watch?v=I7pIf5MSWL4 como motivación. } \\
\checkmark \quad \text { Responde las siguientes preguntas: ¿Qué es sentimos al escuchar el video? } \\
\text { ¿Estoy de acuerdo con el video? } \\
\checkmark \text { Tomando como referencia sus respuestas se vincula con el tema ¿Quiénes } \\
\text { somos en realidad? } \\
\text {-PROCESO } \\
\checkmark \text { Después de ver el video observaremos a nuestra compañera del costado y } \\
\quad \text { colocaremos dos características positivas de ella. } \\
\checkmark \text { Luego las colocaremos en nuestro árbol de vida. } \\
\checkmark \text { Cada una saldrá a exponer las características de su compañera. } \\
\checkmark \text { Trabajaremos en grupo un esquema donde podrán encontrar quiénes son } \\
\text { realmente. } \\
\checkmark \text { Se utilizará la app Termotic que, además de identificar las emociones } \\
\text { básicas, permite indicar la intensidad de lo que sienten, permitiendo } \\
\text { la posibilidad de crear un diario personal en el que registrar las propias } \\
\text { emociones. } \\
\text {-SALIDA } \\
\checkmark \text { Se realizará la retroalimentación sobre lo que se ha observado. } \\
\checkmark \text { Se enseñará una técnica de relajación como cierre. } \\
\checkmark \text { Metacognición: ¿Qué aprendí? ¿Qué pasos realicé para aprenderlo? ¿Qué } \\
\text { dificultades tuve? ¿Cómo las superé? } \\
\text { OBSERVACIONES: De acuerdo con lo que determine la psicóloga, se realizará } \\
\text { el monitoreo de las alumnas. }\end{array}$ & 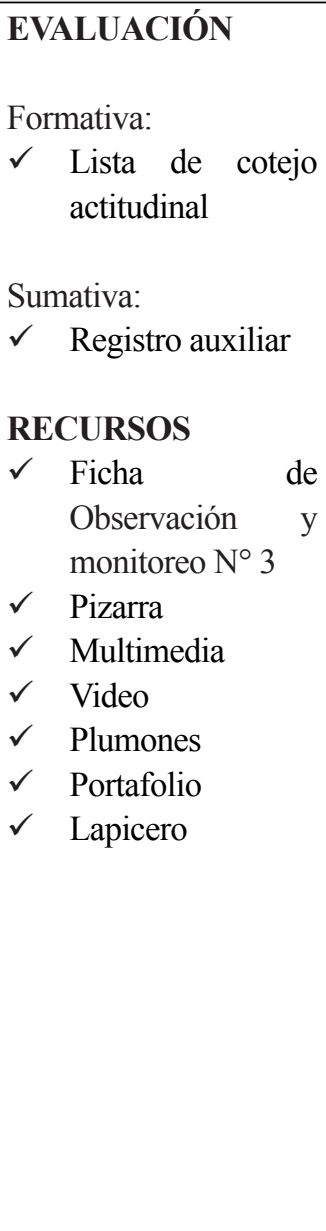 \\
\hline
\end{tabular}

\begin{tabular}{|c|c|c|}
\hline \multicolumn{3}{|c|}{ ACTIVIDADES Y ESTRATEGIAS DE PROGRAMA DE INTERVENCIÓN } \\
\hline $\begin{array}{l}\text { SESIÓN } 4 \\
\text { DURACIÓN: } \\
2 \text { horas }\end{array}$ & $\begin{array}{l}\text { RECONOCIENDO MIS PROBLEMAS } \\
\text { MOMENTOS: } \\
\text {-INICIO } \\
\checkmark \text { Se preguntará cómo se han sentido durante las sesiones y abordaje } \\
\text { terapéutico. } \\
\text {-PROCESO } \\
\checkmark \text { Atiende a la explicación correspondiente del tema, escribe las ideas más } \\
\text { importantes. } \\
\checkmark \text { Realiza trabajo individual de acuerdo a los problemas que presenta, escribe } \\
\quad \text { en un papel y luego comparte con sus compañeras. } \\
\checkmark \text { Trabajaremos en grupo para encontrar alternativas de solución. } \\
\text {-SALIDA } \\
\checkmark \text { Se realizará la retroalimentación sobre lo expuesto. } \\
\checkmark \text { Se realizará un test en Psico Tests. } \\
\checkmark \text { Se enseñará una técnica de relajación como cierre. } \\
\checkmark \text { Metacognición: ¿Qué aprendí? ¿Qué pasos realicé para aprenderlo? ¿Qué } \\
\quad \text { dificultades tuve? ¿Cómo las superé? } \\
\text { OBSERVACIONES: De acuerdo con lo que determine la aplicación se realizará } \\
\text { la terapia. }\end{array}$ & 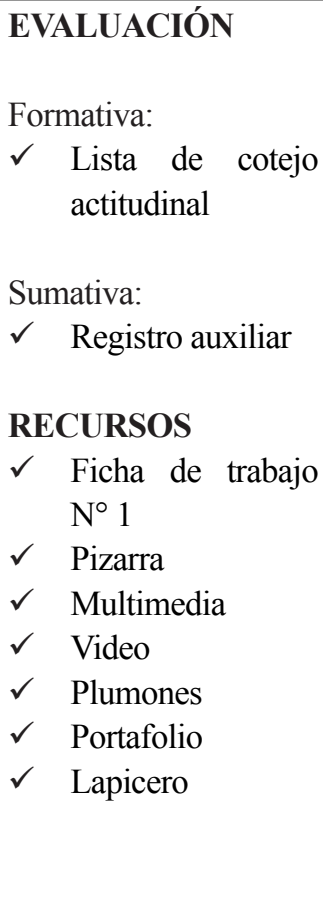 \\
\hline
\end{tabular}


ACTIVIDADES PARA EL TRABAJO CON ALUMNAS Y ALUMNOS DE 1RO. A 5TO. DE SECUNDARIA

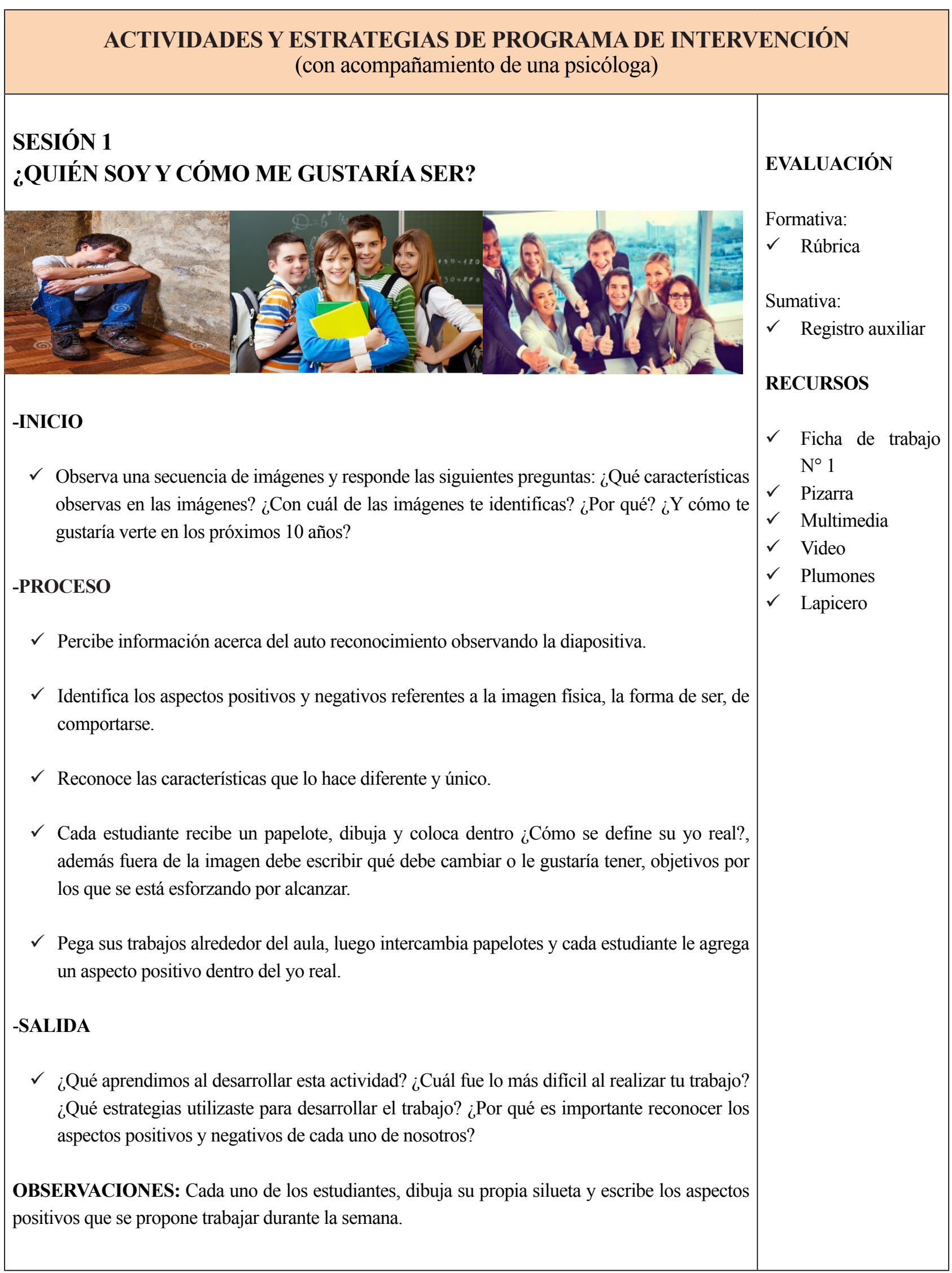




\begin{tabular}{|c|c|c|}
\hline \multicolumn{3}{|c|}{ ACTIVIDADES Y ESTRATEGIAS DE PROGRAMA DE INTERVENCIÓN } \\
\hline $\begin{array}{l}\text { SESIÓN } 2 \\
\text { DURACIÓN: } \\
2 \text { horas }\end{array}$ & $\begin{array}{l}\text { JUGANDO PARA FORTALECER NUESTRAS HABILIDADES } \\
\text { SOCIALES } \\
\text {-INICIO } \\
\checkmark \quad \text { Observa un video acerca de la importancia de las habilidades sociales https:// } \\
\text { www.youtube.com/watch? }=9 \mathrm{bXZQbEUYM} \mathrm{y} \mathrm{responde} \mathrm{las} \mathrm{siguientes} \\
\text { preguntas: ¿Qué son las habilidades sociales? ¿Qué ventajas tienen las } \\
\text { habilidades sociales? ¿Por qué debemos cultivar las habilidades sociales? } \\
\text {-PROCESO } \\
\checkmark \quad \text { Percibe información observando un video sobre la importancia de las } \\
\quad \text { habilidades sociales: https://www.youtube.com/watch?v=TdSSl5gXzIY. } \\
\checkmark \quad \text { Comenta sobre el mensaje que transmite el video. } \\
\checkmark \quad \text { Identifica la importancia del valor de la palabra y expresa sus puntos de vista. } \\
\checkmark \quad \text { Analiza sobre los pensamientos que se debe cambiar y mejorar. } \\
\checkmark \quad \text { Trabajamos en grupo. } \\
\checkmark \quad \text { Se forman grupos de cinco estudiantes y desde su lugar, empiezan a danzar } \\
\checkmark \quad \text { acompañados de un fondo musical. } \\
\text { Cuando la música se detiene abrazan a un integrante del grupo y mencionan } \\
\checkmark \quad \text { una cualidad positiva. } \\
\text { En el siguiente todos se dispersan, y cuando se detenga la música, abrazan a } \\
\text { tres personas que menos conversa con ellos. } \\
\text {-SALIDA } \\
\checkmark \quad \text { ¿Qué aprendimos al desarrollar esta actividad? ¿Cuál fue lo más dificil al } \\
\text { realizar tu trabajo? ¿Qué estrategias utilizaste para desarrollar el trabajo? ¿Por } \\
\text { qué es importante fortalecer las habilidades sociales? } \\
\text { OBSERVACIONES: Cada uno escribe cinco habilidades sociales que se } \\
\text { compromete cultivar en el bimestre. }\end{array}$ & 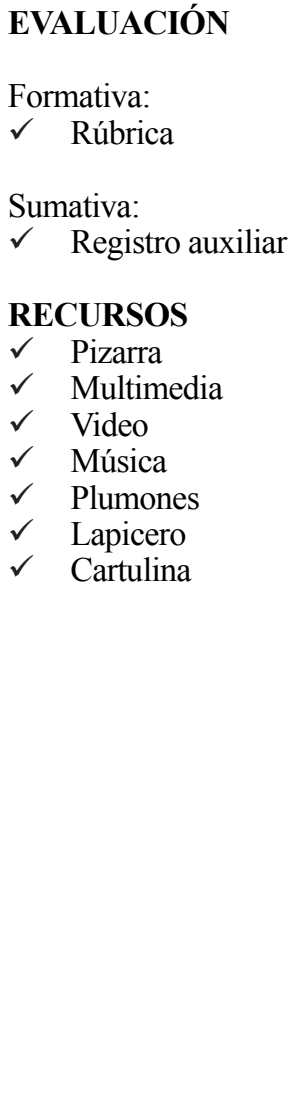 \\
\hline
\end{tabular}

\section{ACTIVIDADES Y ESTRATEGIAS DE PROGRAMA DE INTERVENCIÓN} (con acompañamiento de la psicóloga institucional)

\begin{tabular}{|l|l|}
\hline SESIÓN 3 & VALORANDO A MIS AMIGOS \\
DURACIÓN: & $\begin{array}{l}\text {-INICIO } \\
2 \text { horas }\end{array}$ \\
& $\begin{array}{l}\text { Escucha un fondo musical y el que dirige menciona las siguientes } \\
\text { preguntas: ¿Cómo me siento hoy? ¿Qué debería mejorar? ¿Conozco a mis } \\
\text { amigos? ¿Me valoran como yo quisiera? }\end{array}$
\end{tabular}

\section{-PROCESO}

$\checkmark \quad$ Percibe información acerca de la importancia de tener amigos.

$\checkmark \quad$ Dialoga sobre los momentos más significativos que tuvo con sus amigos más cercanos.

$\checkmark \quad$ Describe las características positivas de sus mejores amigos.

$\checkmark \quad$ Trabajamos en grupos de cinco integrantes.

$\checkmark \quad$ Cada grupo elabora un cartel motivacional que tenga que ver con la valoración de los amigos.

$\checkmark \quad$ Luego nos reunimos, cada grupo comparte el significado del cartel elaborado.

$\checkmark \quad$ Finalmente realizamos un desfile con los carteles elaborados y cantando "Mi

EVALUACIÓN

Formativa:

$\checkmark$ Rúbrica

Sumativa:

$\checkmark \quad$ Registro auxiliar amigo del alma" de Roberto Carlos acompañado del fondo musical https:// www.youtube.com/watch? $\mathrm{v}=\mathrm{hQLjP}-\mathrm{HWabw}$ para ello utilizan su celular para apoyarse con la letra.

\section{-SALIDA}

$\checkmark \quad$ ¿Qué aprendimos al desarrollar esta actividad? ¿Cuál fue lo más difícil al realizar tu trabajo? ¿Qué estrategias utilizastes para desarrollar el trabajo? ¿Por qué es importante fortalecer la amistad entre amigos?

OBSERVACIONES: Cada estudiante elaborará una tarjeta a aquella persona con quien más compartió con ella(él) en este taller para entregarlo en la próxima sesión.

\begin{tabular}{|ll}
\multicolumn{3}{l}{ RECURSOS } \\
$\checkmark$ & Pizarra \\
$\checkmark$ & Multimedia \\
$\checkmark$ & Video \\
$\checkmark$ & Música \\
$\checkmark$ & Plumones \\
$\checkmark$ & Lapicero \\
$\checkmark$ & Cartulina \\
& \\
& \\
& \\
\end{tabular}




\section{ACTIVIDADES PARA EL TRABAJO CON LOS PADRES DE FAMILIA DE LA INSTITUCIÓN EDUCATIVA}

Dirigidas a los Padres de Familia de la Institución Educativa

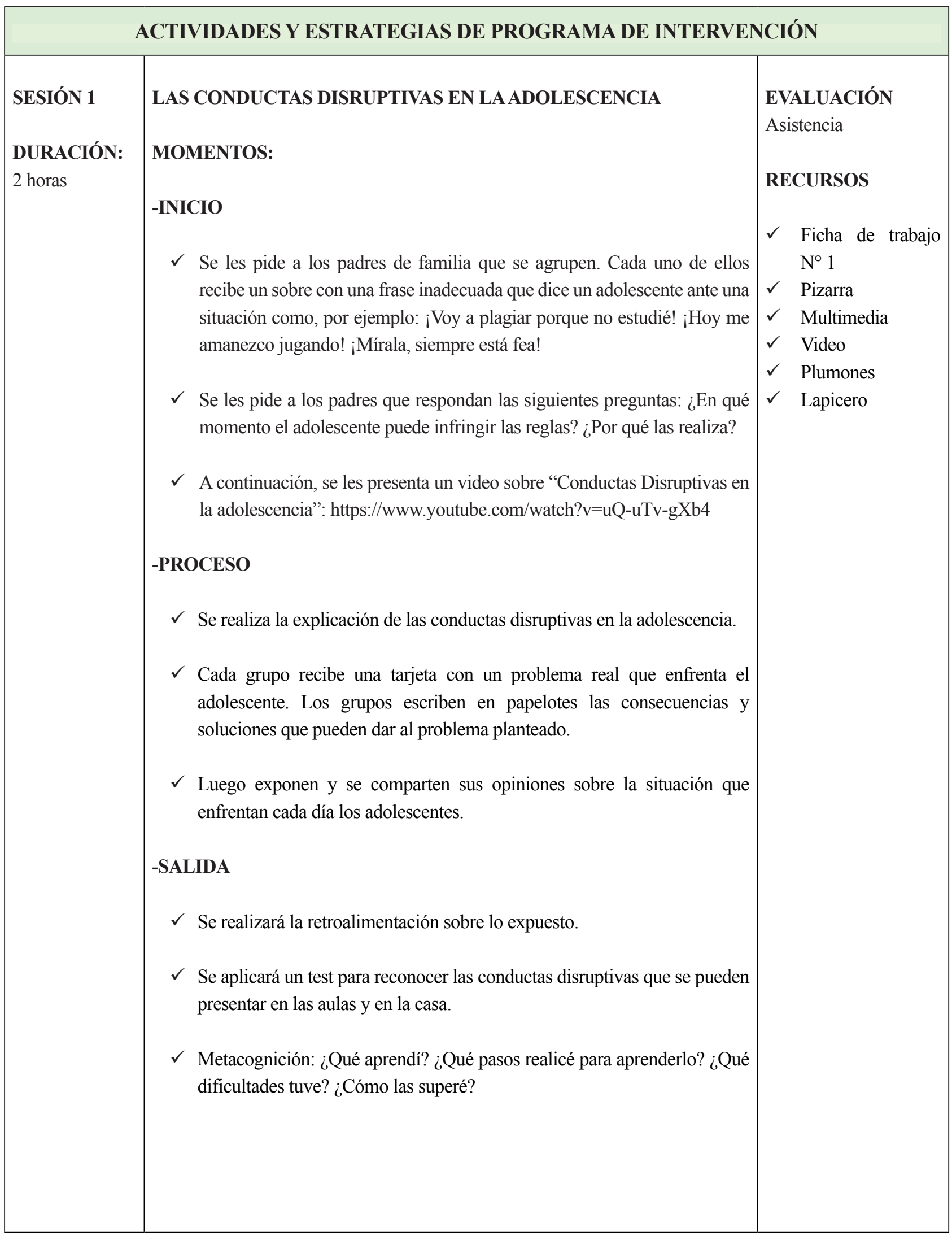




\section{ACTIVIDADES Y ESTRATEGIAS DE PROGRAMA DE INTERVENCIÓN}

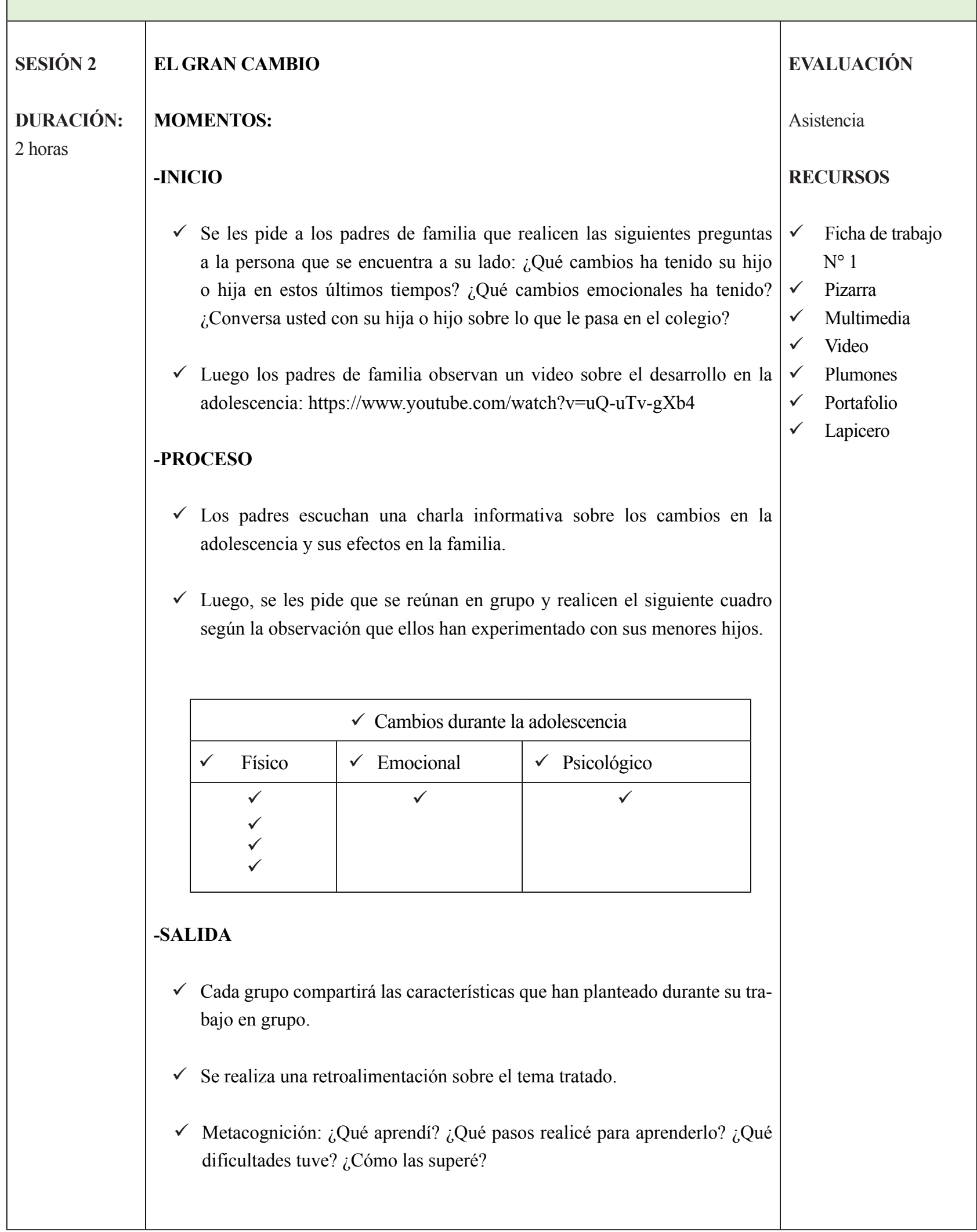




\section{ACTIVIDADES Y ESTRATEGIAS DE PROGRAMA DE INTERVENCIÓN}

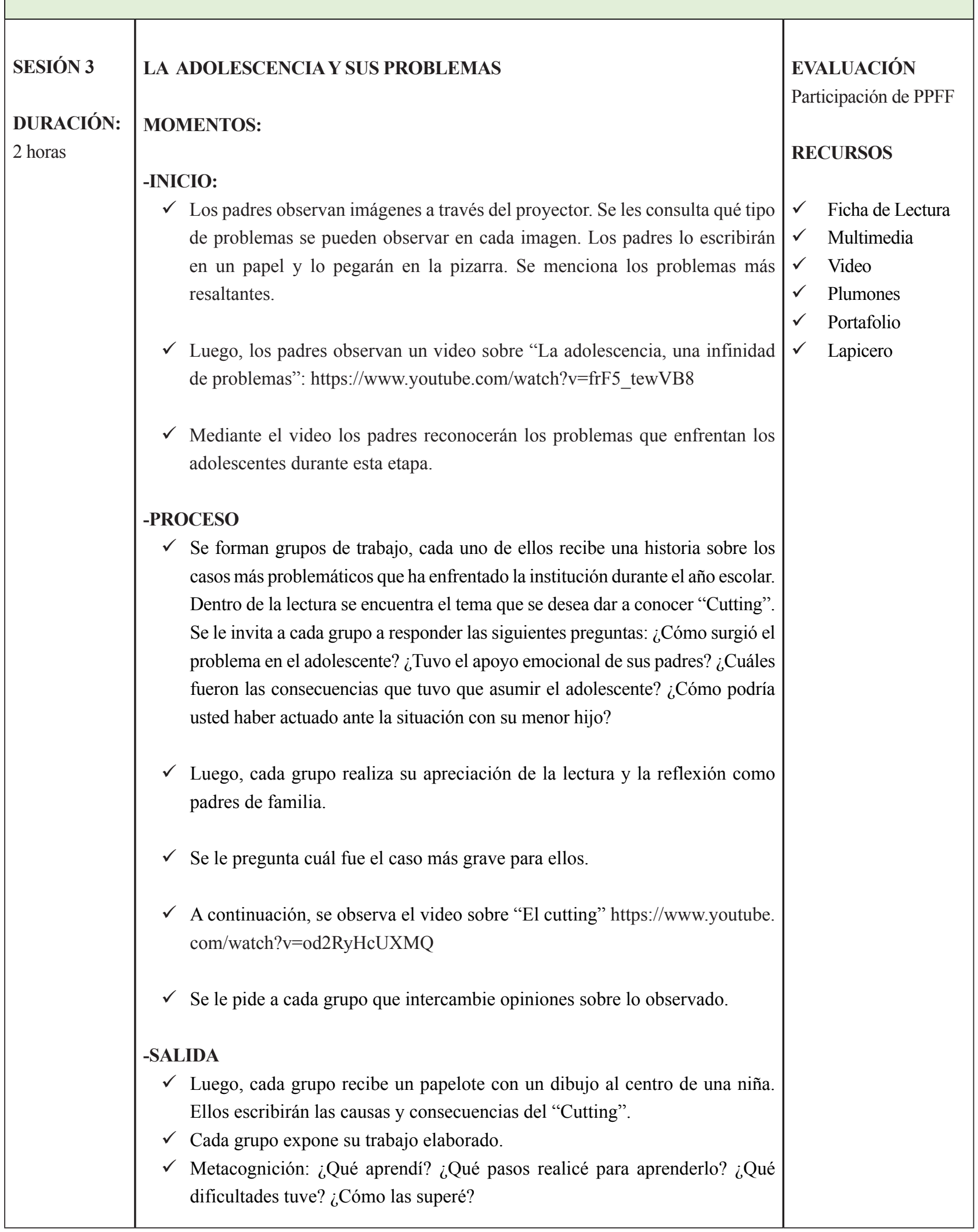


ACTIVIDADES PARA EL TRABAJO CON LOS PADRES DE FAMILIA AFECTADOS

\begin{tabular}{|c|c|c|}
\hline \multicolumn{3}{|c|}{ ACTIVIDADES Y ESTRATEGIAS DE PROGRAMA DE INTERVENCIÓN } \\
\hline $\begin{array}{l}\text { SESIÓN 1 } \\
\text { DURACIÓN: } \\
90 \text { minutos }\end{array}$ & 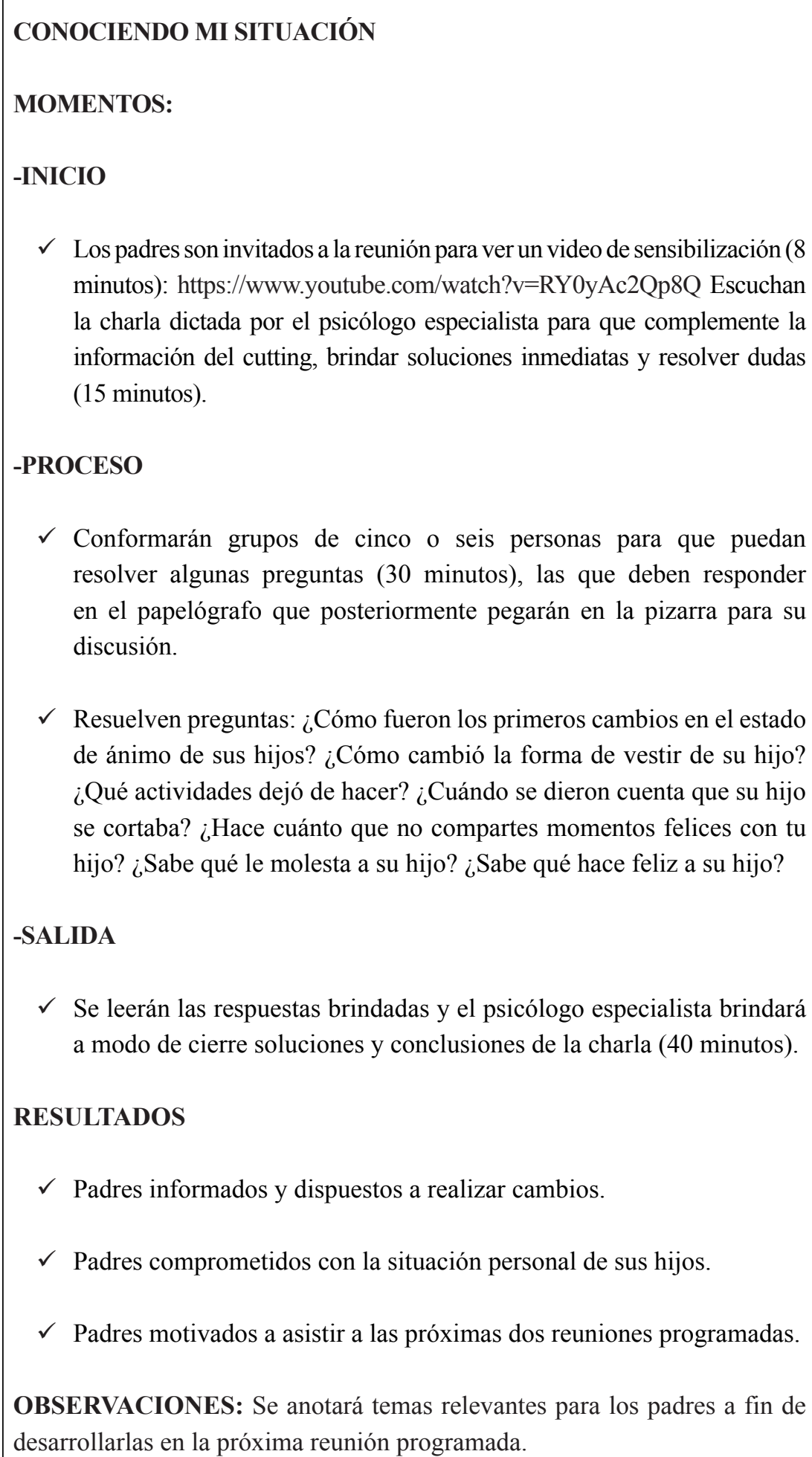 & \begin{tabular}{|ll} 
RECURSOS \\
$\checkmark$ & Ficha de trabajo N \\
& 1 \\
$\checkmark$ & Pizarra \\
$\checkmark$ & Multimedia \\
$\checkmark$ & Video \\
$\checkmark$ & Plumones \\
$\checkmark$ & Portafolio \\
$\checkmark$ & Lapicero
\end{tabular} \\
\hline
\end{tabular}




\begin{tabular}{|c|c|c|}
\hline \multicolumn{3}{|c|}{ ACTIVIDADES Y ESTRATEGIAS DE PROGRAMA DE INTERVENCIÓN } \\
\hline $\begin{array}{l}\text { SESIÓN } 2 \\
\text { DURACIÓN: } \\
90 \text { minutos }\end{array}$ & 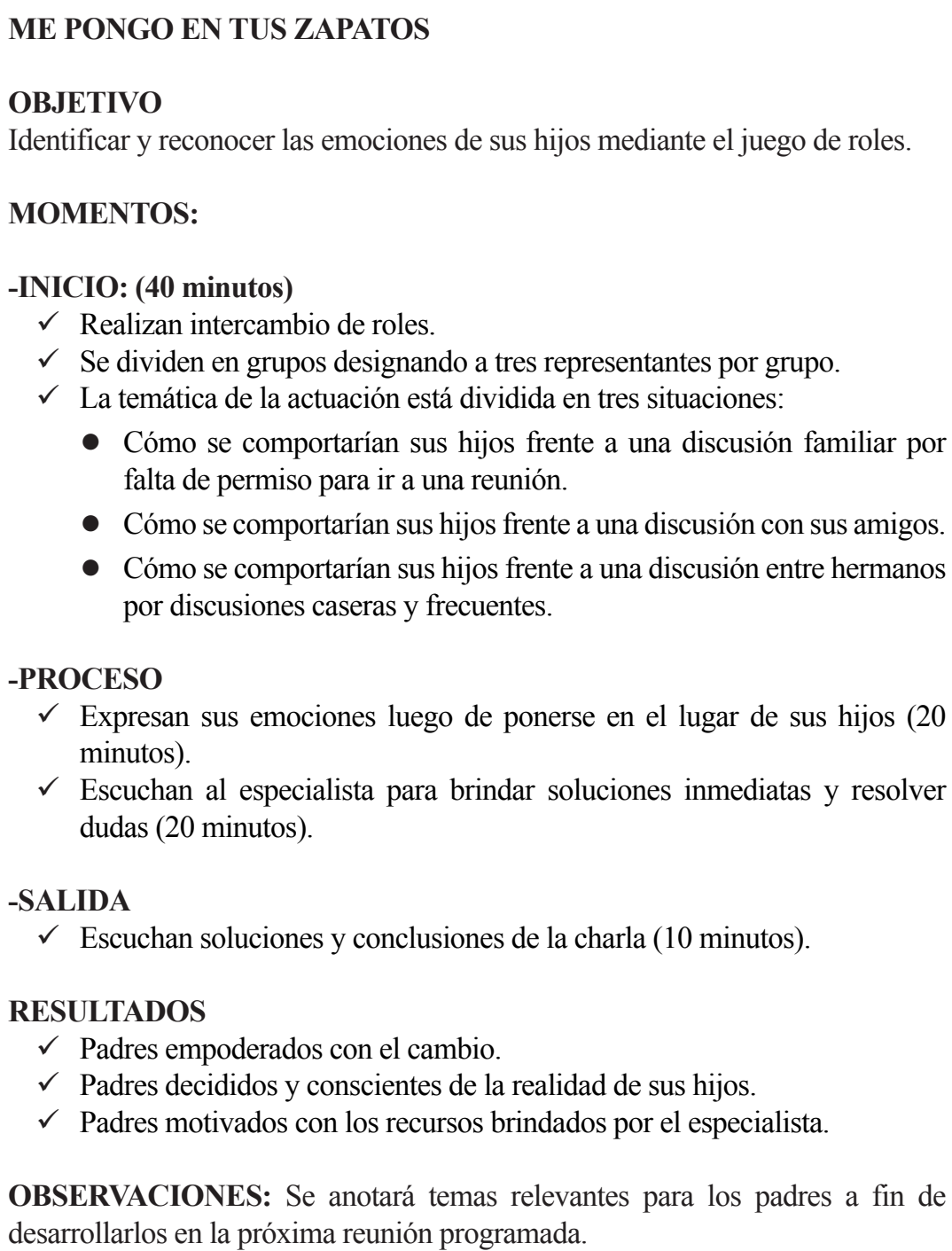 & $\begin{array}{ll}\text { RECURSOS } \\
\checkmark & \text { Pizarra } \\
\checkmark & \text { Multimedia } \\
\checkmark & \text { Plumones } \\
\checkmark & \text { Lapicero }\end{array}$ \\
\hline
\end{tabular}

\begin{tabular}{|c|c|c|}
\hline \multicolumn{3}{|c|}{ ACTIVIDADES Y ESTRATEGIAS DE PROGRAMA DE INTERVENCIÓN } \\
\hline $\begin{array}{l}\text { SESIÓN } 3 \\
\text { DURACIÓN: } \\
60 \text { minutos }\end{array}$ & $\begin{array}{l}\text { EL CAMBIO EMPIEZA YA } \\
\text { MOMENTOS: } \\
\text {-INICIO (30 minutos) } \\
\quad \checkmark \text { Expresan los primeros cambios ejecutados en casa. } \\
\checkmark \quad \text { Comparten ideas de solución entre ellos. } \\
\text {-PROCESO y SALIDA ( } 20 \text { minutos) } \\
\quad \checkmark \text { Escuchan soluciones finales y consejos por parte del psicólogo especialista. } \\
\text { RESULTADOS } \\
\checkmark \text { Padres empoderados con el cambio. } \\
\checkmark \text { Padres motivados con los recursos brindados por el especialista. }\end{array}$ & $\begin{array}{ll}\text { RECURSOS } \\
\checkmark & \text { Pizarra } \\
\checkmark & \text { Multimedia } \\
\checkmark & \text { Plumones }\end{array}$ \\
\hline
\end{tabular}




\begin{tabular}{|c|c|c|}
\hline ACTIVIDADES & OBJETIVOS & RESPONSABLE \\
\hline \multicolumn{3}{|l|}{ Estudiantes afectados } \\
\hline Sesión 1: Libera tus emociones & Reconocer las emociones que les hace daño. & \multirow{4}{*}{$\begin{array}{c}\text { Tutores de grado } \\
\text { Psicólogo especialista } \\
\text { Psicólogo de la institución }\end{array}$} \\
\hline Sesión 2: Me saco la mochila & $\begin{array}{l}\text { Eliminar emociones negativas (ira, miedo, } \\
\text { dolor, tristeza y angustia). }\end{array}$ & \\
\hline Sesión 3: ¿Quién eres en realidad? & $\begin{array}{l}\text { Conocernos a nosotros mismos resaltando las } \\
\text { características positivas de las personas. }\end{array}$ & \\
\hline $\begin{array}{l}\text { Sesión 4: Reconociendo mis } \\
\text { problemas }\end{array}$ & $\begin{array}{l}\text { Identifico mis problemas y les brindo } \\
\text { soluciones. }\end{array}$ & \\
\hline \multicolumn{3}{|l|}{ Estudiantes no afectados } \\
\hline $\begin{array}{l}\text { Sesión 1: ¿Quién soy y cómo me } \\
\text { gustaría ser? }\end{array}$ & $\begin{array}{l}\text { Identificar los aspectos positivos y negativos } \\
\text { para reconocerse un ser valioso. }\end{array}$ & \multirow{3}{*}{$\begin{array}{l}\text { Tutores de grado } \\
\text { Psicólogo de la institución }\end{array}$} \\
\hline $\begin{array}{l}\text { Sesión 2: Jugando para fortalecer } \\
\text { nuestras habilidades }\end{array}$ & Fortalecer sus habilidades sociales. & \\
\hline Sesión 3: Valorando a mis amigos & Valorar la importancia de tener amigos. & \\
\hline
\end{tabular}

\section{MATRIZ LÓGICA}

\begin{tabular}{|c|c|c|}
\hline ACTIVIDADES & OBJETIVOS & RESPONSABLE \\
\hline \multicolumn{3}{|l|}{ Padres de las estudiantes afectadas } \\
\hline Sesión 1: Conociendo mi situación & $\begin{array}{l}\text { Brindar información macro y micro de la } \\
\text { problemática a nivel local y personal. }\end{array}$ & \multirow[t]{3}{*}{$\begin{array}{l}\text { Psicólogo de la institución } \\
\text { Psicólogo especialista }\end{array}$} \\
\hline Sesión 2: Me pongo en tus zapatos & $\begin{array}{l}\text { Indicar y reconocer a sus hijos mediante el } \\
\text { intercambio de juego de roles. }\end{array}$ & \\
\hline Sesión 3: El cambio empieza ya & $\begin{array}{l}\text { Brindar soluciones a través de la reflexión de } \\
\text { los padres. }\end{array}$ & \\
\hline \multicolumn{3}{|l|}{ Padres de los estudiantes no afectados } \\
\hline Sesión 1: El gran cambio & $\begin{array}{l}\text { Informar a los padres de familia sobre los } \\
\text { cambios psicológicos, biológicos y físicos que } \\
\text { suceden durante esta etapa. }\end{array}$ & \multirow[t]{3}{*}{$\begin{array}{c}\text { Tutores } \\
\text { Psicólogo de la institución }\end{array}$} \\
\hline $\begin{array}{l}\text { Sesión 2: La adolescencia y sus } \\
\text { problemas }\end{array}$ & $\begin{array}{l}\text { Identificar los problemas que afectan al } \\
\text { adolescente en esta etapa. }\end{array}$ & \\
\hline Sesión 3: Conductas disruptivas & $\begin{array}{l}\text { Identificar e informar sobre las conductas } \\
\text { agresivas que suceden en esta etapa. } \\
\text { Dar a conocer estrategias para el } \\
\text { reconocimiento de conductas disruptivas en } \\
\text { casa. }\end{array}$ & \\
\hline
\end{tabular}




\section{REFERENCIAS}

Castro (2014). Autolesión no suicida en adolescentes peruanas: Una aproximación diagnóstica y psicopatológica. Recuperado de: http://www.scielo.org.pe/pdf/rnp/v77n4/ a05v77n4.pdf

Cruz (2006). La autolesión, una visión desde la psicoterapia humanista integrativa. Recuperado de: http://bonding.es/laautolesion-una-vision-desde-la-psicoterapiahumanista-integrativa/

D’Arcy Lyness (Junio de 2012). Psicólogos en Red. Obtenido de "Cutting" (Autoflagelo). Recuperado de: http://www.psicologosenred. com/index.php?option=com_content $\&$ view $=$ article $\&$ id $=134$ : cutting-autoflagelo\&catid $=82 \&$ Itemid $=470$

Del Olmo, M. (2005). Prejuicios y estereotipos: un replanteamiento de uso y utilidad como mecanismos sociales. XXI Revista de Educación, pp. 13-23.

Diario Expreso ec. (8 de noviembre de 2014). Expreso.ec. Obtenido de cutting. Una "moda" peligrosa en adolescentes. Recuperado de: http://expreso.ec/historico/ el-cutting-unamoda-peligrosa-enadolescentes-JEGR 7285006

Flores, Cancino, Figueroa (2018). Revisión sistemática sobre conductas autolesivas sin intención suicida en adolescentes. Revista Cubana de Salud Pública. 2018, 44(4).

Naranjo Pereira, M. L. (3 de diciembre de 2007). Autoestima: un factor relevante en la vida de la persona y tema esencial del proceso educativo. Revista Electrónica Actualidades Investigativas en Educación, VII(3), pp. 2-3.

Fecha de presentación: 23-10-2019

Fecha de aceptación: 07-11-2019 